\title{
Effect of a Pharmacologically Induced Decrease in Core Temperature in Rats Resuscitated from Cardiac Arrest
}

\author{
Laurence M. Katz, M.D., \\ Department of Emergency Medicine, University of North Carolina School of Medicine \\ Jonathan E. Frank, M.S., \\ Department of Emergency Medicine, University of North Carolina School of Medicine \\ Lawrence T. Glickman, V.M.D., PhD., \\ Department of Emergency Medicine, University of North Carolina School of Public Health \\ Gerald McGwin Jr, PhD., \\ Epidemiology, University of Alabama at Birmingham \\ Brice H. Lambert, MA., and \\ Epidemiology, University of Alabama at Birmingham \\ Christopher J. Gordon, PhD. \\ Neurotoxicology Branch, US Environmental Protection Agency, Research Triangle Park, NC
}

\section{Abstract}

\begin{abstract}
Aim-Hypothermia is recommended by international guidelines for treatment of unconscious survivors of cardiac arrest to improve neurologic outcomes. However, temperature management is often underutilized because it may be difficult to implement. The present study evaluated the efficacy of pharmacologically induced hypothermia on survival and neurological outcome in rats resuscitated from cardiac arrest.
\end{abstract}

Methods-Cardiac arrest was induced for 10 minutes in 120 rats. Sixty-one rats were resuscitated and randomized to normothermia, physical cooling or pharmacological hypothermia 5 minutes after resuscitation. Pharmacological hypothermia rats received a combination of ethanol, vasopressin and lidocaine (HBN-1). Physical hypothermia rats were cooled with intravenous iced

\footnotetext{
(C) 2015 Published by Elsevier Ireland Ltd.

Correspondence: Laurence M. Katz, Department of Emergency Medicine, CB 7594, 170 Manning Dr. Physician Office Building First Floor, Chapel Hill, North Carolina 27599, Fax 919-966-3047, office 919-966-6442, 1katz@ med.unc.edu.

Publisher's Disclaimer: This is a PDF file of an unedited manuscript that has been accepted for publication. As a service to our customers we are providing this early version of the manuscript. The manuscript will undergo copyediting, typesetting, and review of the resulting proof before it is published in its final citable form. Please note that during the production process errors may be discovered which could affect the content, and all legal disclaimers that apply to the journal pertain.

Disclaimer: The research described in this article has been reviewed by the National Health and Environmental Effects Research Laboratory, U.S. Environmental Protection Agency, and approved for publication. Approval does not signify that the contents necessarily reflect the views and policies of the Agency, nor does the mention of trade names of commercial products constitute endorsement or recommendation for use.
}

Conflict of Interest Statement

Dr. Katz submitted a patent entitled Methods and Compositions for the Induction of Hypothermia and owns stock in the University of North Carolina spin-off company Hibernaid. No other author has a conflict of interest. 
saline and cooling pads. Rats in the pharmacological hypothermia group received $\mathrm{HBN}-1$ at ambient temperature $\left(20^{\circ} \mathrm{C}\right)$. Normothermic rats were maintained at $37.3 \pm 0.2^{\circ} \mathrm{C}$.

Results-HBN-1 ( $\mathrm{p}<0.0001)$ shortened the time $(85 \pm 71$ minutes) to target temperature $\left(33.5^{\circ} \mathrm{C}\right)$ versus physical hypothermia $(247 \pm 142$ minutes). The duration of hypothermia was 17.0 \pm 6.8 hours in the HBN-1 group and $17.3 \pm 7.5$ hours in the physical hypothermia group $(p=0.918)$. Survival $(p=0.034)$, neurological deficit scores $(p<0.0001)$ and Morris Water Maze performance after resuscitation ( $\mathrm{p}=0.041$ ) was improved in the HBN-1 versus the normothermic group. HBN-1 improved survival and early neurological outcome compared to the physical hypothermia group while there was no significant difference in performance in the Morris water maze.

Conclusion-HBN-1 induced rapid and prolonged hypothermia improved survival with good neurological outcomes after cardiac arrest suggesting that pharmacologically-induced regulated hypothermia may provide a practical alternative to physical cooling.

Targeted temperature management is recommended to reduce brain damage after resuscitation from cardiac arrest in humans although the optimal target temperature remains controversial. ${ }^{1-4}$ The American Heart Association (AHA) and the International Liaison Committee on Resuscitation include hypothermia in their guidelines for treatment of patients resuscitated from cardiac arrest. Despite these guidelines, therapeutic hypothermia is underutilized with less than $12 \%$ of hospitals in the US using this potentially lifesaving approach. ${ }^{5,6}$ The most often cited reason for not using therapeutic hypothermia is "it is too technically difficult." 5

Physical methods to forcefully lower body temperature include ice bags, cooling pads and endovascular devices. Such cooling methods can be inefficient in lowering core body temperature of adult subjects because of naturally occurring thermoregulatory responses consisting of shivering, cutaneous vasoconstriction and increased metabolism. ${ }^{7}$ As a result, drugs such as opiates and paralytics are often given before initiation of physical cooling methods. ${ }^{8}$ Furthermore, these drugs add to the complexity and safety of cooling because they may induce hypotension, altered mental status, respiratory arrest, and the need for mechanical ventilation. In addition, the equipment and drugs required for induction of physical hypothermia are not always available pre-hospital, thus delaying the initiation of cooling and time to target temperature which may influence neurological outcome. ${ }^{9}$

HBN-1 was developed as a pharmacological alternative to physical methods to lower core temperature and induce a regulated state of therapeutic hypothermia. ${ }^{10,11}$ Regulated hypothermia is thought to be operative by lowering the body's temperature set point in the hypothalamic regulatory center. ${ }^{12}$ When the temperature set point is lowered, the body responds by reducing metabolic rate, blocks shivering, and increases heat loss through peripheral vasodilatation and sweating. HBN-1 is a patented pharmaceutical preparation that combines ethanol, vasopressin and lidocaine. ${ }^{10}$ It induces a rapid and prolonged hypothermia even at room temperature without the need for chemical paralysis, sedation, ancillary equipment, or mechanical ventilation. ${ }^{10}$ The drug is administered intravenously so paramedics could use it in the field, thus minimizing delays in inducing hypothermia. 
The purpose of this study was to evaluate the effect of HBN-1 on body temperature, mortality and neurological outcome in rats resuscitated from cardiac arrest. We hypothesized that survival with good neurological outcome following pharmacologicallyinduced hypothermia would be significantly different compared to normothermia or physically-induced cooling.

\section{Materials and Methods}

The Institutional Animal Care and Use Committee approved the study in accord with National Institutes of Health Guidelines. Sprague-Dawley female rats weighing 300-350 grams were resuscitated from asphyxial cardiac arrest and then randomized to normothermic, physical hypothermic or pharmacological hypothermic (HBN-1) groups. We prospectively assigned a treatment group to rats after resuscitation from cardiac arrest by picking a piece of paper (12 with normothermia, 12 with physical hypothermia and 12 with HBN-1) out of an opaque jar with the group assignment on the paper. If the rat survived 15 days, that assignment would be removed from further assignment. If the rat did not survive the 15 days, the data from that rat would be analyzed, but the assignment was placed $b$ ack in the jar to assure that 12 rats would be available for Morris Water Maze analysis in each group upon completion of the study. Rats in the normothermic group had their core body temperature maintained at a target temperature of $37.3^{\circ} \mathrm{C}$ before asphyxial cardiac arrest and during reperfusion. Rats in the physical hypothermic group were wrapped in a cooling pad set to $4^{\circ} \mathrm{C}$ (Medivance, Arctic Sun, Louisville, $\mathrm{CO}$ ) and infused with iced saline $\left(4^{\circ} \mathrm{C}\right)$ starting 5 minutes after return of spontaneous circulation (ROSC) and continued for 12 hours after resuscitation. Hypothermia was maintained in the physical hypothermic group by having the core temperature signal from a surgically implanted telemetric probe (MiniMitter, Bend, OR) control a servo-regulated incubator (Brinsea, Titusville, FL), the cooling pad, and a fan. Rats in the pharmacological hypothermic group received room temperature $\mathrm{HBN}-1$ (ethanol $3.03 \mathrm{~g} / \mathrm{kg}$, vasopressin $0.13 \mathrm{U} / \mathrm{kg}$, lidocaine $3.2 \mathrm{mg} / \mathrm{kg}$ ) infused intravenously at ambient temperature $\left(20^{\circ} \mathrm{C}\right)$ starting 5 minutes after ROSC and continued for 12 hours after resuscitation. All three groups received an initial $30 \mathrm{ml} / \mathrm{kg}$ bolus of fluid (normal saline at room temperature in the normothermic group and normal saline at $4{ }^{\circ} \mathrm{C}$ in the physical hypothermic group) initiated 5 minutes after ROSC at a rate of $60 \mathrm{ml} / \mathrm{kg} / \mathrm{hr} \times 30$ minutes followed by $1.5 \mathrm{ml} / \mathrm{kg} / \mathrm{hr}$ for 12 hours. The time to target temperature was defined as the time from initiation of cooling until a core temperature of $33.5^{\circ} \mathrm{C}$ was attained. All rats were prepared for asphyxial cardiac arrest and reperfusion as previously described. ${ }^{13}$ Briefly, rats were anesthetized with $4 \%$ isoflurane, intubated and mechanically ventilated with a combination of $30 \%$ oxygen and $70 \%$ nitrous oxide and wrapped in a thermal blanket to approximate the mass and thermal inertia of a larger mammal. ${ }^{14}$ Rats were then covered with Arctic Sun pads (turned off in the normothermic and HBN-1 groups), and titrated isoflurane anesthesia was maintained throughout the surgery. Catheters were placed in a femoral vessel to monitor mean arterial blood pressure, for blood draws, and for administration of intravenous drugs. Blood samples and cardiovascular parameters were recorded at baseline and at 10 minutes, at 30 minutes, and at 30 minute intervals thereafter, until 180 minutes after ROSC. A telemetric temperature probe (Data Sciences International, St Paul, MN) was inserted and secured to the posterior peritoneum, behind the liver via a 
midline laparotomy. Rats were chemically paralyzed with vecuronium $(1 \mathrm{mg} / \mathrm{kg})$ intravenously, and apneic asphyxia was induced by interrupting ventilation. Asphyxia led to cardiac arrest within 4 minutes in all rats, and asphyxia was maintained for 10 minutes. Rats were resuscitated with intravenous epinephrine $(0.008 \mathrm{mg} / \mathrm{kg})$, sodium bicarbonate $(1 \mathrm{mEq} /$ $\mathrm{kg}$ ), mechanical ventilation with $100 \%$ oxygen and chest compressions. Chest compressions were stopped when there was ROSC (mean arterial pressure greater than $60 \mathrm{mmHg}$ for more than 5 minutes) or no ROSC after 2 minutes. Rats were extubated 180 minutes after ROSC, the arterial line was removed and the venous line was tunneled through to the shoulder, externalized and connected to a tether which allowed the rats unrestricted movement. Rats had free access to food and water during recovery. A neurological deficit score was performed daily for 10 days after ROSC by an investigator blinded to therapeutic interventions. The rat neurological deficit score tested cranial nerve function, coordination (balance beam walk, placing test, depth perception, righting reflex), motor and sensory function. ${ }^{13}$ On days 11-15 after ROSC, rats were trained in a Morris Water Maze with four swimming sessions a day by a research assistant blinded to intervention. The rats were placed in one of four entrance quadrants (north, south, east or west) of the pool in random order and swam until they found the hidden platform or 90 seconds had elapsed without finding the platform. If a rat was unable to find the hidden platform, they were placed on the hidden platform for 30 seconds. Rats learn to find the hidden platform by referencing the location of illuminated figures on the side of the pool relative to the hidden platform. ${ }^{15}$ The average time over four swimming trials required to locate the hidden platform (latency time) was compared between groups. After completion of Morris Water Maze testing rats were euthanized with an overdose of isoflurane.

\section{Statistical analysis}

Physiological variables, neurological deficit scores and latency time were reported as means and standard deviations and compared between groups at specific time point(s) using analysis of variance. Latency time across days was also compared between groups by a repeated measure ANOVA. Kaplan-Meier curves were used to evaluate survival in the groups and the difference was compared using a log rank test. P-values $<0.05$ were considered statistically significant. All statistical analysis was performed using SAS v 9.3 (SAS Institute, Cary, NC).

\section{Results}

Fifty-one percent of 120 rats were successfully resuscitated following induction of asphyxial cardiac arrest. Physiological variables at baseline were comparable between groups. There was no difference in time to cardiac arrest or ROSC between groups. The time from ROSC to a target temperature (TTT) of $33.5^{\circ} \mathrm{C}$ was shorter with $\mathrm{HBN}-1$ ( $85 \pm 71$ minutes) compared to physical hypothermia ( $247 \pm 142$ minutes) ( $\mathrm{p}<0.0001)$ (Figure 1$)$. The duration of hypothermia was $17.0 \pm 6.8$ hours in the HBN-1 group and $17.3 \pm 7.5$ hours in the physical hypothermic group $(\mathrm{p}=0.918)$ (Figure 1).

Mean arterial pressure (MAP) was higher in the $\mathrm{HBN}-1$ group at 10 minutes $(\mathrm{p}=0.04)$ and 30 minutes $(\mathrm{p}<0.0001)$ after resuscitation compared to the other groups (Figure 2$)$. 
A total of $12 / 24,12 / 24$ and 12/1 3 resuscitated rats survived 15 days in the normothermic, physical hypothermic and HBN-1 groups, respectively. HBN-1 infused after resuscitation from cardiac arrest improved survival compared to normothermia and physical hypothermia $(\mathrm{p}=0.034)($ Figure 3$)$.

HBN-1 and physical hypothermia reduced Neurological Deficit Scores compared to the normothermic group. $(\mathrm{p}<0.0001)$ (Figure 4)

The HBN-1 group had a lower mean Neurological Deficit Score compared to physical hypothermia group for the first four days after resuscitation, but from day five onwards the mean Neurological Deficit Scores for these two groups were not significantly different. (Figure 4).

Performance in the Morris Water Maze evaluates spatial memory and learning. Shorter latency times are associated with improved spatial memory and learning. ${ }^{15}$ The decrease in latency time was greater in each hypothermic group compared to normothermia for each passing day ( $\mathrm{p}=0.041$ ). (Figure 5). HBN-1 latency on day 13 and day 15 after resuscitation from cardiac arrest was significantly shorter compared to normothermia on comparable days $(\mathrm{p}=0.027$ and $\mathrm{p}=0.017$ respectively) (Figure 5). Latency time in the HBN-1 group was comparable to latency with physical hypothermia $(\mathrm{p}=0.184)$.

\section{Discussion}

HBN-1 administered intravenously at room temperature in rats resuscitated from cardiac arrest shortened the time to target temperature when compared to the physical cooling method to induce hypothermia. A continuous i nfusion of HBN-1 for 12 hours maintained a state of therapeutic hypothermia for over 12 hours. In addition, when compared to normothermia, rats with HBN-1 induced hypothermia showed improved survival with good neurological outcome measured by a Neurological deficit score and testing of spatial memory and learning in the Morris Water Maze. In addition, HBN-1 induced hypothermia improved survival and was associated with an improvement in early neurological outcome when compared to physical hypothermia, but there was no significant difference in Morris Water Maze testing. The early improvement in neurological outcome of HBN-1 compared to physical cooling was not expected and we plan to conduct further studies designed to explore the reasons for this observation.

HBN-1 induced a transient rise in MAP during early reperfusion when compared to the other two treatment groups. There is preclinical evidence that flow promotion in the setting of hypothermia may improve neurological outcome. ${ }^{16}$ However, there are no prospective clinical trials supporting a survival advantage of flow promotion methods or vasopressin alone after resuscitation from cardiac arrest. In addition, a meta-analysis by Layek ${ }^{17}$ suggested that vasopressin during cardiopulmonary resuscitation had no beneficial effect on ROSC, survival or neurological outcome in an unselected patient population, so it is unlikely that vasopressin alone was the reason HBN treated animals had a survival advantage.

All rats in the current study were insulated to simulate larger mammals and the time to target temperature in the physical hypothermia groups was comparable to times reported in human 
clinical trials. ${ }^{18}$ The rate of cooling was not controlled, but was a dependent variable determined by the method of cooling (physical versus pharmacological with HBN-1). The study design does not allow for determination of the relative contribution of HBN-1, independent of the shorter time to target temperature produced by HBN-1, on neurological outcome. However, pilot experiments (data not shown) in HBN-1 rats exploring increasing environmental temperature above ambient to prolong the time to target temperature comparable to physical hypothermia rats resulted in no survivors. This was not unexpected since aggressive warming during reperfusion worsens neurological outcome. ${ }^{19}$ The therapeutic window of opportunity after cardiac arrest has not been well defined, but may be inversely proportional to the duration of cerebral ischemia. ${ }^{20,} 21$ Pharmacological cooling with HBN-1 may provide a method for reaching target temperature within a narrower therapeutic window.

Therapeutic hypothermia may be underutilized because physical cooling methods require sedation, paralysis, and mechanical cooling devices to implement. ${ }^{6} \mathrm{HBN}-1$, a combination of generic drugs that induce regulated hypothermia, pharmacologically lowers body temperature without the need for chemical paralysis, sedation, or physical cooling methods. The neural mechanism of action of HBN-1 is not known. However, based on a rat's behavioral and autonomic thermoregulatory responses, it is likely associated with stimulation of heat loss pathways and/or suppression of heat production/conservation pathways in CNS thermoregulatory centers of the pre-optic and anterior hypothalamus. ${ }^{10,22}$ Intravenous administration of a drug such as HBN-1 that lowers body temperature through a reset of a thermostat in the hypothalamus is a simple approach for inducing and maintaining therapeutic hypothermia. It has the potential to overcome perceived barriers to implementation of therapeutic hypothermia in a field or clinical setting.

There is a disconnect between drugs that demonstrate neuroprotection in the laboratory and efficacy in clinical trials. ${ }^{23}$ These translational failures are multifactorial, including limitations of individual animal models to reflect the complexity of drug targets for preventing reperfusion injury. ${ }^{24}$ The outcome model of cardiac arrest used in the present study attempts to address some limitations recognized in previous animal models. ${ }^{13}$ Thermoregulation is also an appealing translational target of neuroprotectant agents because reperfusion injury is temperature dependent across species. Human clinical trials have confirmed the value of thermoregulation to improve survival while preserving neurological function, although the optimal target temperature has been challenged. ${ }^{1,2,4}$

Unexpected toxicity is a common reason for failure of many neuroprotectant drugs. ${ }^{25}$ HBN-1 is a combination of commercially available generic drugs, each having good safety profiles. Drug induced hypotension has previously been reported as a reason for translational failure of preclinical neuroprotectant drugs. ${ }^{26-28}$ No hypotension was noted with administration of HBN-1 after resuscitation from cardiac arrest in the present study. However, more extensive toxicology studies are needed to evaluate the safety of the combination of drugs that comprise $\mathrm{HBN}-1$ before consideration for clinical use.

There were several limitations to the study. First, it was not designed to determine the relative contributions of the individual components in HBN-1 to survival or neurological 
outcome. However, to our knowledge, there are no published human clinical trials to suggest a neuroprotective effect for the individual components. Second, rats were wrapped with a thermal blanket to simulate the mass and thermal inertia of larger mammals. That is a relatively simplistic approach for replicating the complicated thermal exchanges that occur in humans. ${ }^{14}$ However, wrapping the rats produced a temperature curve in the physical cooling group similar to that produced by physical cooling in humans. ${ }^{18}$ Third, the study was not designed to evaluate the relative contributions of HBN-1 induced shortened time to target temperature or transient rise in blood pressure during reperfusion on survival. Future studies that control these variables would be valuable to determine the relative contribution of these important physiological variables to survival. Fourth, no brain histology was performed on the rats in the present study. Brain pathology provides an objective measure of neuronal damage, but is not always a reliable predictor of neurological function. ${ }^{29}$ Fifth, HBN-1 improved neurological outcome 15 days after resuscitation, but longer outcome studies are required to establish permanence of the benefit. Despite these limitations, two separate neurological outcome assessments were measured (including a neurological deficit score and water maze activity). The results suggest that HBN-1 improved survival with good neurological function after cardiac arrest and complement earlier results ${ }^{11}$ of reduced reperfusion injury as measured by brain biomarkers (CSF and serum Neuron Specific Enolase) in large animals that received HBN-1 during early reperfusion from cardiac arrest.

\section{Conclusions}

Intravenous infusion of HBN-1 may provide a practical pharmacological alternative to physical cooling to decrease and maintain core body temperature after resuscitation from cardiac arrest. Infusion of HBN-1 maintained therapeutic hypothermia for a prolonged period of time in rats and improved survival with good neurological function. Clinical trials in patients following ROSC are required to confirm these findings.

\section{Supplementary Material}

Refer to Web version on PubMed Central for supplementary material.

\section{Acknowledgment}

The authors would like to thank Alex Finch and Jonathan Pozner for the care of the animals, surgical assistance and data collection.

Funding Source

This study was funded by grant 1R21NS064103-01A1 from the National Institute of Neurological Disorders and Stroke.

\section{References}

1. HACA Study Group. Mild therapeutic hypothermia to improve the neurologic outcome after cardiac arrest. N Engl J Med. 2002; 346:549-556. [PubMed: 11856793]

2. Bernard SA, Gray TW, Buist MD, et al. Treatment of comatose survivors of out-of-hospital cardiac arrest with induced hypothermia. N Engl J Med. 2002; 346:557-563. [PubMed: 11856794] 
3. Lopez-de-Sa E, Rey JR, Armada E, et al. Hypothermia in comatose survivors from out-of-hospital cardiac arrest: Pilot trial comparing 2 levels of target temperature. Circulation. 2012; 126:28262833. [PubMed: 23136160]

4. Nielsen N, Wetterslev J, Cronberg T, et al. Targeted temperature management at 33 degrees c versus 36 degrees c after cardiac arrest. N Engl J Med. 2013; 369:2197-2206. [PubMed: 24237006]

5. Merchant RM, Soar J, Skrifvars MB, et al. Therapeutic hypothermia utilization among physicians after resuscitation from cardiac arrest. Crit Care Med. 2006; 34:1935-1940. [PubMed: 16691134]

6. Jena AB, Romley JA, Newton-Cheh C, Noseworthy P. Therapeutic hypothermia for cardiac arrest: Real-world utilization trends and hospital mortality. J Hosp Med. 2012; 7:684-689. [PubMed: 23023977]

7. Frank SM, Higgins MS, Fleisher LA, et al. Adrenergic, respiratory, and cardiovascular effects of core cooling in humans. Am J Physiol. 1997; 272:R557-R562. [PubMed: 9124478]

8. Sessler DI. Defeating normal thermoregulatory defenses: Induction of therapeutic hypothermia. Stroke. 2009; 40:e614-e621. [PubMed: 19679849]

9. Sendlebach S, Hearst MO, Johnson PJ, et al. Effects on variation in temperature management on cerebral performance scores in patients who received therapeutic hypothermia post cardiac arrest. Resuscitation. 2012; 83:829-834. [PubMed: 22230942]

10. Katz LM, Frank J, McGwin G Jr, et al. Induction of a prolonged hypothermic state by druginduced reduction in the thermoregulatory set-point. Ther Hypothermia Temp Manag. 2012; 2:6166. [PubMed: 23667774]

11. Katz LM, McGwin G Jr, Gordon CJ. Drug induced therapeutic hypothermia after asphyxial cardiac arrest in swine. Ther Hypothermia Temp Manag. 2012; 2:176-182. [PubMed: 24716490]

12. Gordon CJ. The therapeutic potential of regulated hypothermia. Emerg Med J. 2001; 18:81-89. [PubMed: 11300205]

13. Katz L, Ebmeyer U, Safar P,et al. Outcome model of asphyxial cardiac arrest in rats. J Cereb Blood FlowMetab. 1995; 15:1032-1039.

14. Cohen ML. Measurement of the thermal properties of human skin. A review. J Invest Dermatol. 1977; 69:333-338. [PubMed: 894075]

15. Morris R. Developments of a water-maze procedure for studying spatial learning in the rat. J Neurosci Methods. 1984; 11:47-60. [PubMed: 6471907]

16. Safar P, Xiao F, Radovsky, et al. Improved cerebral resuscitation from cardiac arrest in dogs with mild hypothermia plus blood flow promotion. Stroke. 1996; 27:105-113. [PubMed: 8553385]

17. Layek A, Maitra S, Pal S, et al. Efficacy of vasopressin during cardio-pulmonary resuscitation in adult patients: A meta-analysis. Resuscitation. 2014; 85:855-863. [PubMed: 24704138]

18. Kim F, Nichol G, Maynard C, et al. Effect of prehospital induction of mild hypothermia on survival and neurological status among adults with cardiac arrest: A randomized clinical trial. JAMA. 2014; 311:45-52. [PubMed: 24240712]

19. Dietrich WD, Bramlett HM. Hyperthermia and central nervous system injury. Prog Brain Res. 2007; 162:201-217. [PubMed: 17645921]

20. Ye S, Weng Y, Sun S, et al. Comparison of the durations of mild therapeutic hypothermia on outcome after cardiopulmonary resuscitation in the rat. Circulation. 2012; 125:123-129. [PubMed: 22086880]

21. Oddo M, Ribordy V, Feihl F, et al. Early predictors of outcome in comatose survivors of ventricular fibrillation and non-ventricular fibrillation cardiac arrest treated with hypothermia: A prospective study. Crit Care Med. 2008; 36:2296-2301. [PubMed: 18664785]

22. Fehlner KS, Gordon CJ. Effect of ambient temperature on thermoregulation in rats following preoptic/anterior hypothalamic injection of physostigmine. Neuropharmacology. 1985; 24:993997. [PubMed: 4069323]

23. O'Collins VE, Macleod MR, Donnan GA, et al. 1,026 experimental treatments in acute stroke. Ann Neurol. 2006; 59:467-477. [PubMed: 16453316]

24. Kahle MP, Bix GJ. Successfully climbing the "stairs": Surmounting failed translation of experimental ischemic stroke treatments. Stroke Res Treat. 2012; 2012 
25. DiMasi JA, Feldman L, Seckler A, Wilson A. Trends in risks associated with new drug development: Success rates for investigational drugs. Clin Pharmacol Ther. 2010; 87:272-277. [PubMed: 20130567]

26. ECC Committee, Subcommittees and Task Forces of the American Heart Association. 2005 American Heart Association guidelines for cardiopulmonary resuscitation and emergency cardiovascular care. Circulation. 2005; 112:IV1-IV203. [PubMed: 16314375]

27. Randomized clinical study of thiopental loading in comatose survivors of cardiac arrest. Brain Resuscitation Clinical Trial I study group. N Engl J Med. 1986; 314:397-403. [PubMed: 2868412]

28. A randomized clinical study of a calcium-entry blocker (lidoflazine) in the treatment of comatose survivors of cardiac arrest. Brain Resuscitation Clinical Trial II study group. N Engl J Med. 1991; 324:1225-1231. [PubMed: 2014035]

29. Shamy JL, Buonocore MH, Makaron LM, et al. Hippocampal volume is preserved and fails to predict recognition memory impairment in aged rhesus monkeys (macaca mulatta). Neurobiol Aging. 2006; 27:1405-1415. [PubMed: 16183171] 
Core Temperature After Resuscitation from Cardiac Arrest

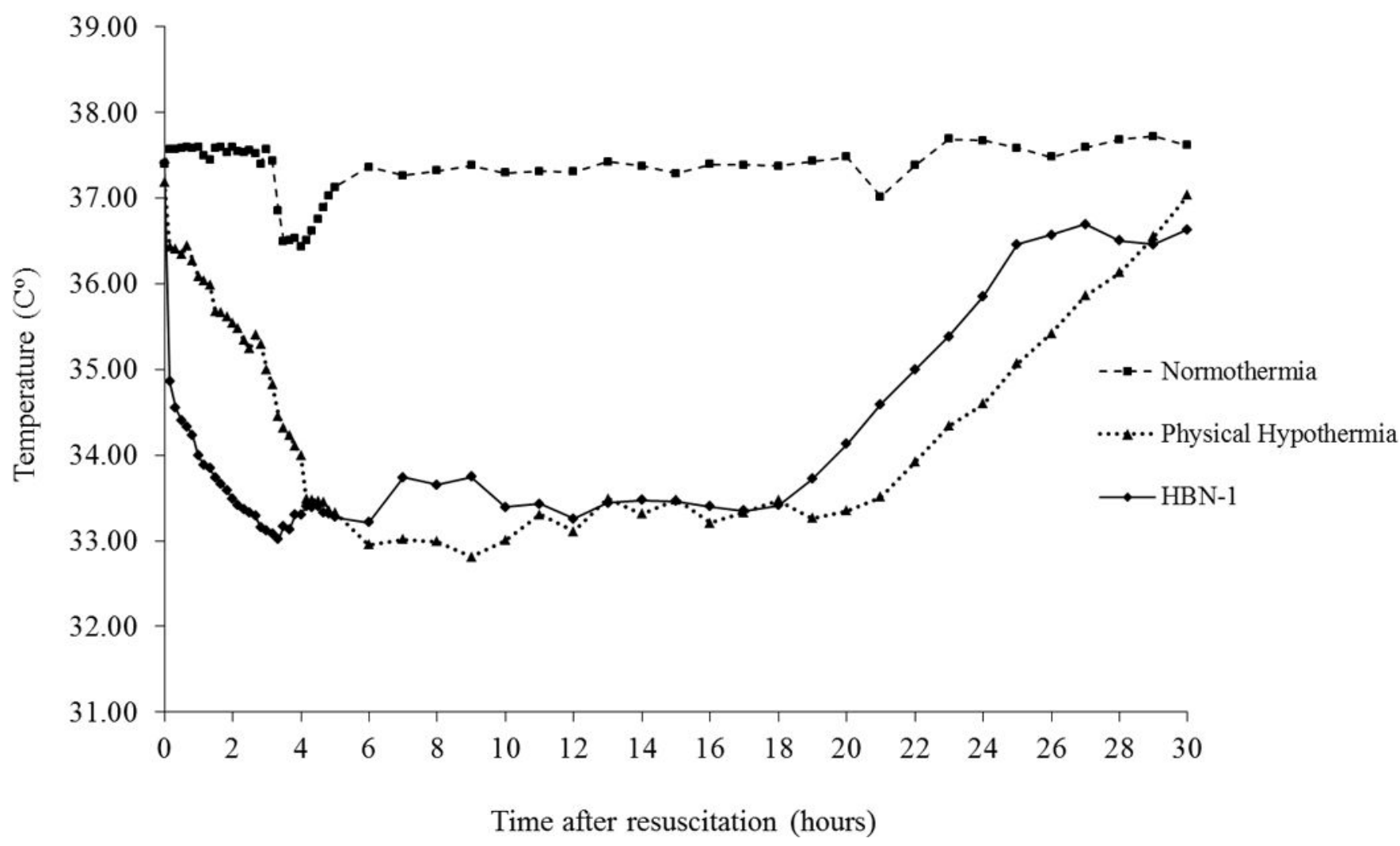

Figure 1.

Core temperature after resuscitation from cardiac arrest. Time in hours after resuscitation. Physical hypothermia and pharmacological hypothermia (HBN-1) were induced 5 minutes after ROSC and maintained for 12 hours. 


\section{Mean Arterial Blood Pressure (MAP)}

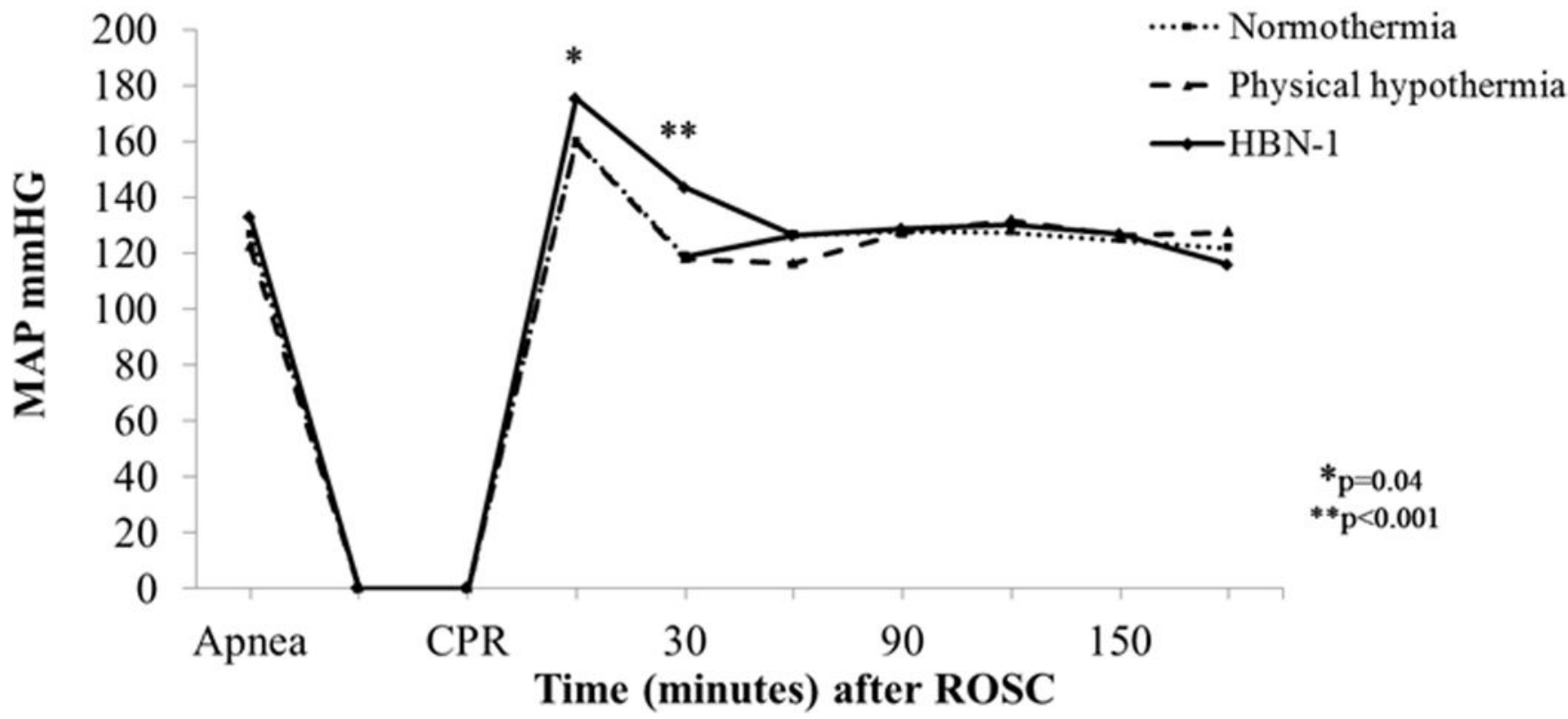

Figure 2.

Mean arterial blood pressure (MAP) at onset of insult (apnea), cardiac arrest (CA), resuscitation (CPR) and during reperfusion. Physical hypothermia and pharmacological hypothermia (HBN-1) were induced 5 minutes after ROSC and maintained for 12 hours. 


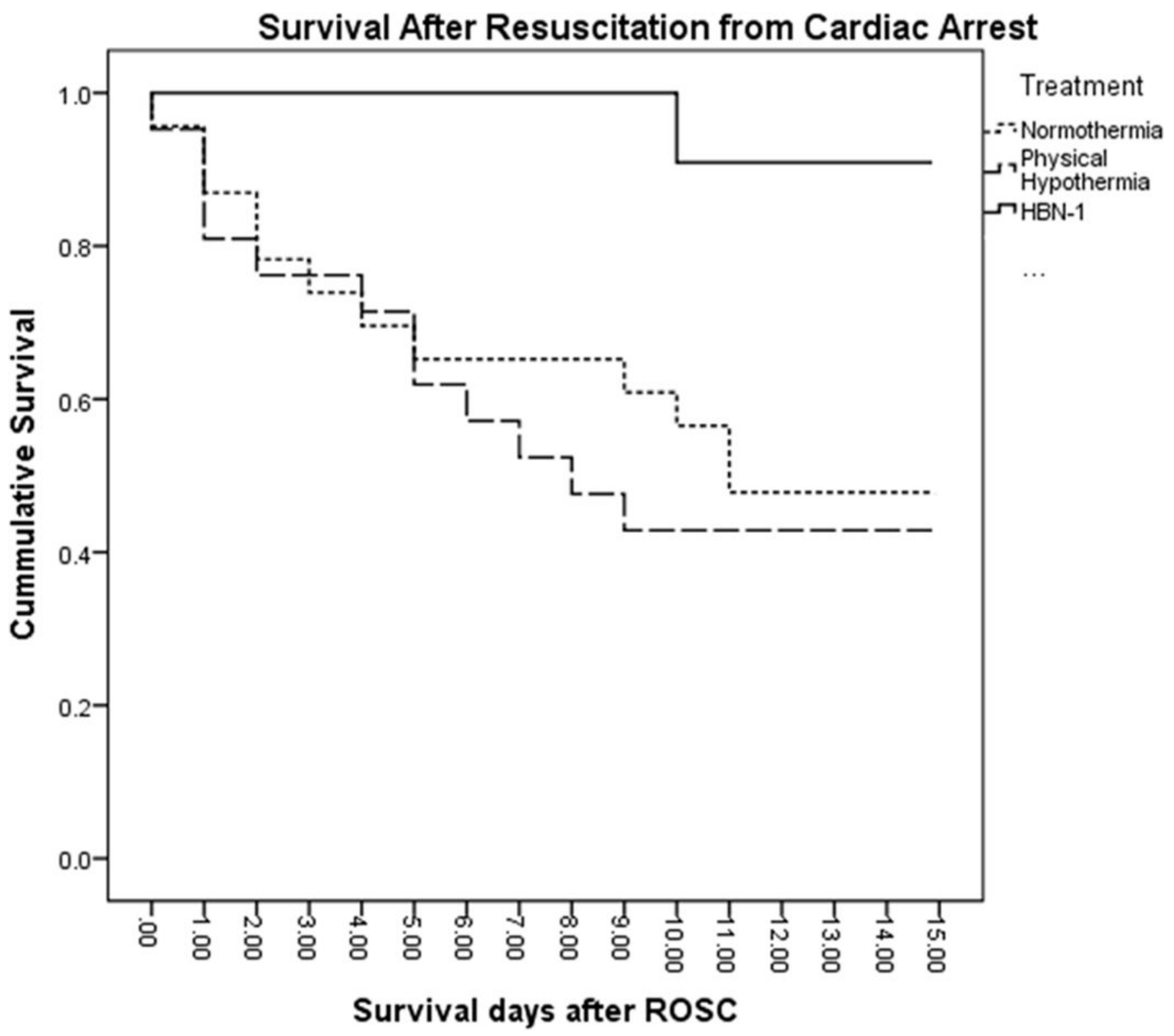

Figure 3.

Cumulative survival curves. Physical hypothermia and pharmacological hypothermia (HBN-1) were induced 5 minutes after ROSC and maintained for 12 hours. 


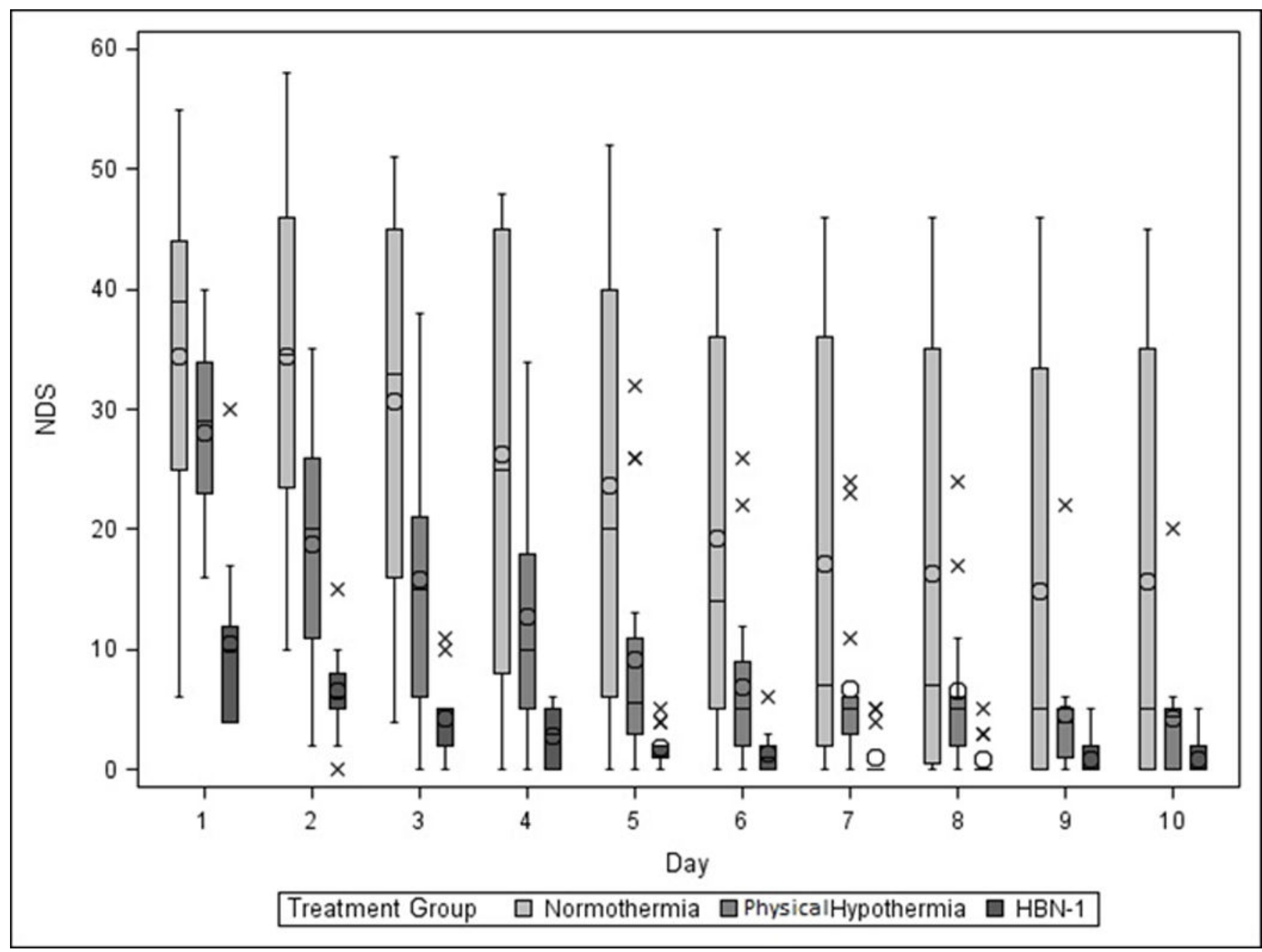

Figure 4.

Median Neurological deficit score (NDS) in the normothermic, physical hypothermia and pharmacological hypothermia (HBN-1) groups on days 1 through 10 after resuscitation from cardiac arrest. Physical and pharmacological hypothermia were induced 5 minutes after ROSC and maintained for 12 hours. Circles are the means, lines are the medians and x's are the outliers. Error bars represent standard deviations. 


\section{Morris Water Maze Latency time after cardiac arrest}

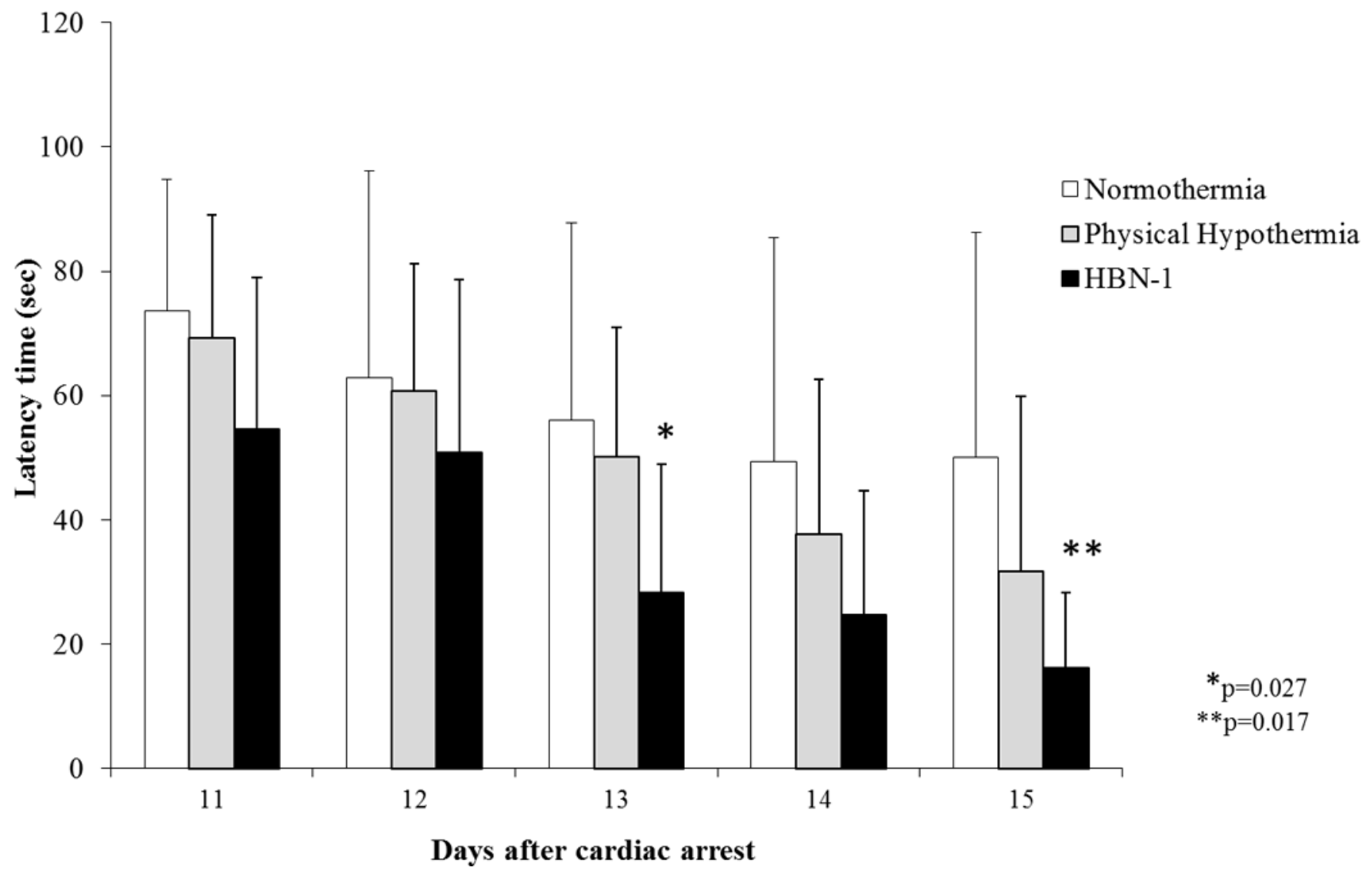

Figure 5.

Morris Water Maze Performance after cardiac arrest. The mean time to find the hidden platform (latency time) in rats treated with normothermia, physical hypothermia or pharmacological hypothermia (HBN-1) therapy initiated 5 minutes after resuscitation from cardiac arrest and maintained for 12 hours. Error bars represent standard deviations. *,** Compared to the normothermic group. 